\title{
BMJ Open Factors other than medical acuity that influence hospitalisation: a scoping review protocol
}

\author{
Mellena Giday, ${ }^{1,2}$ Meghaan Hawes, ${ }^{1,2}$ Ann Madhavan, ${ }^{3}$ Maralyssa Bann ${ }^{1,2}$
}

To cite: Giday M, Hawes M, Madhavan A, et al. Factors other than medical acuity that influence hospitalisation: a scoping review protocol. BMJ Open 2019;9:e028949. doi:10.1136/ bmjopen-2019-028949

- Prepublication history and additional material for this paper are available online. To view these files, please visit the journal online (http://dx.doi. org/10.1136/bmjopen-2019028949).

Received 4 January 2019 Revised 21 March 2019 Accepted 17 May 2019

\section{Check for updates}

C Author(s) (or their employer(s)) 2019. Re-use permitted under CC BY-NC. No commercial re-use. See rights and permissions. Published by BMJ.

${ }^{1}$ Division of General Internal Medicine/Hospital Medicine, Harborview Medical Center, Seattle, Washington, USA ${ }^{2}$ Department of Medicine, University of Washington School of Medicine, Seattle, Washington, USA

${ }^{3}$ Health Sciences Library, University of Washington, Seattle, Washington, USA

Correspondence to Dr Mellena Giday; mellenag@uw.edu

\begin{abstract}
Introduction There is evidence that patients are admitted to the hospital with low-acuity medical issues, though delineation of the underlying factors has not been comprehensively explored. This scoping review will provide an overview of the existing literature regarding factors outside of acute medical illness that influence hospitalisation of adults. The review will also seek to provide a review of common language and definitions used in the research on this phenomenon.

Methods and analysis The scoping review framework, outlined by Arksey and 0'Malley and expanded on by Levac et al, will be used as the basis for this study. A systematic search of seven databases (PubMed, CINAHL, PsycINFO, EMBASE, Web of Science, Sociological Abstracts and Social Science Abstracts) will be conducted to identify existing literature followed by a standardised two-phase, two-reviewer process to select relevant papers for inclusion. Relevant studies will investigate adult nonpsychiatric hospital admission plus at least one additional factor unrelated to medical acuity. Details of the work will be extracted, including the terminology used and perspectives included. An assessment of methodological quality will be performed using a tool designed for mixedmethods systematic review.

Ethics and dissemination The scoping review protocol delineates a transparent and rigorous review process, the results of which will be disseminated through peerreviewed publication and presentation at relevant local or national meetings. The study does not require ethics approval as the data will be accumulated through the review of published, peer-reviewed literature and grey literature.
\end{abstract}

\section{BACKGROUND}

Severity of illness risk stratification scores do not always correlate well with risk of hospitalisation, ${ }^{1}$ particularly when used in marginalised populations. ${ }^{2}$ That patients with low acuity of medical illness are admitted to the hospital $^{3}$ suggests the risk of hospitalisation is not determined solely by acuity of illness. Indeed, in one report, up to $51 \%$ of hospitalisations were strongly or moderately influenced by a factor other than medical acuity. ${ }^{4}$

Operationalising the drivers of hospitalisation that are not specifically related to medical

\section{Strengths and limitations of this study}

This review will establish a baseline understanding of the influence of factors other than medical acuity on the decision to hospitalise adult patients.

- A broad, systematic search strategy has been designed in collaboration with a research librarian.

- Quality of studies included will be appraised and reported.

- The aim to incorporate disparate language, perspectives and research methodologies may reveal widely divergent lines of evidence that do not easily synthesise into a unified conceptual framework.

- Only articles published in English will be included.

acuity has been fragmented and problematic in the literature. This body of work lacks standard nomenclature, using terms such as 'non-medical factors', 'deprivation, ${ }^{5}$ 'social factors, ${ }^{6}$ 'patient characteristics ${ }^{7}$ or naming specific factors such as homelessness, ${ }^{8}$ food insecurity ${ }^{10}$ or comorbidity of medical, psychiatric and social conditions. ${ }^{11}$

\section{STUDY RATIONALE AND OBJECTIVES}

We aim to examine the association of factors other than medical acuity with hospitalisation. As part of our analysis, we will map the nomenclature used to define these factors, as well as research methodologies used, perspectives investigated and study results identified. By doing this, we intend to unite several bodies of work. The function of this product will be to provide a comprehensive review of the evidence base in order to identify any inconsistent use of terminology, to inform clinicians making admission decisions and policymakers or funders who review admission decisions and to expose gaps in the knowledge.

\section{METHODS AND ANALYSIS}

The review will follow a standard framework for scoping reviews as delineated by Arksey 
and $\mathrm{O}^{\prime}$ Malley ${ }^{12}$ and expanded on by Levac et al. ${ }^{13}$ This process is comprised of the following stages: (1) identifying the research question; (2) identifying relevant studies; (3) selecting studies; (4) charting the data; (5) collating, summarising and reporting the results; and an optional (6) consultation. The six stages are discussed in detail below.

\section{Stage 1: identifying the research questions}

Because scoping reviews are intended to synthesise available evidence on a topic, the initial research question may remain broad. Levac et al suggest clarifying the focus of inquiry by identifying the purpose and intended outcome of the study.

For this review, the overarching research question being asked is: what has been studied about the relationship between factors other than medical acuity and hospitalisation of adults?

The following subquestions have been identified by study researchers to guide this work:

- What terminology is used to define factors other than medical acuity?

- Which factors have been investigated with respect to impact on hospitalisation (and, by default, which have not been investigated)?

- Whose perspective on factors other than medical acuity and hospitalisation has been investigated? (patient, physician, nurse, social worker and so on).

- What type of research on factors other than medical acuity and hospitalisation has been conducted? (epidemiological, cohort, qualitative/narrative and so on).

- What findings have been reported? Are there any factors consistently associated with hospitalisation?

\section{Stage 2: identifying relevant studies}

The second stage of the proposed scoping review will identify relevant studies for selection and data extraction. We will conduct comprehensive searches of seven databases (PubMed, CINAHL, PsycINFO, EMBASE, Web of Science, Sociological Abstracts and Social Science Abstracts). In collaboration with a medical librarian, we have developed a PubMed search strategy (see online supplementary file) utilising medical subject headings and free-text terms that retrieves 5240 results. The PubMed search has been modified to fit the database search requirements for the remaining databases. All searches will be limited to results in English involving adult subjects. No date limits will be applied. As we explore the search question, further iteration of the search may occur and will be captured by the review process. Search results will be imported into Covidence, an online systematic review management programme, and duplicates will be removed prior to the selection process.

\section{Stage 3: selecting studies}

Arksey and O'Malley suggest that the selection of studies based on inclusion and exclusion criteria may take place post hoc after sufficient familiarity with the literature is established. Levac proposes that the research team should determine study exclusion and inclusion criteria at the outset of the process, with the ability to refine the search strategy and review additional articles for inclusion if warranted.

As recommended, two reviewers will independently select all relevant studies; in cases of disagreement, a third reviewer will provide input. A two-level selection process will be used. First, articles will be screened by study title and abstract against the inclusion and exclusion criteria listed below and then read in full to ensure appropriateness for study inclusion.

\section{Inclusion criteria}

For studies to be included, they should meet the following criteria:

- Adult patient population.

- Includes hospital admission of any status (observation or inpatient status).

- Includes assessment of some additional factor other than medical acuity, including but not limited to: socioeconomic status, insurance status, barriers to healthcare access, lack of housing, food insecurity, mental health disorder, cognitive difficulties, physical disability or limitation, immigration status, non-English speakers.

- Empiric studies of any design type.

- Published either in peer-reviewed journals or in the 'grey' literature (conference proceedings, preprints and so on).

\section{Exclusion criteria}

Studies with the following criteria will be excluded:

- Not published in English.

- Primarily study inpatient psychiatric hospital admission.

- Commentaries, consensus statements or other non-empiric reports.

The process of study selection will be reported using a Preferred Reporting Items for Systematic Reviews and Meta-Analyses (PRISMA) flowchart. ${ }^{14}$

\section{Stage 4: charting the data}

Data will be extracted from each selected article using a standardised extraction template. Detailed description of data extraction fields is available in table 1.

Overarching domains will include article characteristics, study design, characterisation of factor other than medical acuity and findings. Based on initial literature review, we predict that some studies will use collective or general terminology for factors unrelated to medical acuity (eg, 'non-medical-acuity', social factors or deprivation') while other studies will use specific, named factors such as homelessness. We will extract terminology used in either category as well as any definitions provided in the study for the terms used. 


\begin{tabular}{ll}
\hline Table 1 Data extraction template \\
\hline Domain/subdomains & Description \\
\hline $\begin{array}{l}\text { Article characteristics } \\
\text { Author }\end{array}$ & $\begin{array}{l}\text { Who was the first author of this } \\
\text { study? }\end{array}$ \\
\hline Journal & $\begin{array}{l}\text { In what peer-reviewed journal } \\
\text { was this study published? }\end{array}$ \\
Year & $\begin{array}{l}\text { In what year was this study } \\
\text { published? }\end{array}$ \\
Country & $\begin{array}{l}\text { Which country is this article } \\
\text { from? }\end{array}$ \\
Publication type & $\begin{array}{l}\text { Is this a journal article, } \\
\text { conference abstract, grey } \\
\text { literature? }\end{array}$
\end{tabular}

\begin{tabular}{|c|c|}
\hline \multicolumn{2}{|l|}{ Study design } \\
\hline Study design & What was the study design? \\
\hline Objective & $\begin{array}{l}\text { What was the stated study } \\
\text { objective? }\end{array}$ \\
\hline Population & $\begin{array}{l}\text { What population was studied? } \\
\text { Reference inclusion/exclusion } \\
\text { criteria. }\end{array}$ \\
\hline Methodology & $\begin{array}{l}\text { What methods were used in the } \\
\text { study? }\end{array}$ \\
\hline Outcome & What outcome was reported? \\
\hline Perspective investigated & $\begin{array}{l}\text { Was the perspective of the } \\
\text { patient, family/surrogate, } \\
\text { physician, nurse, social worker } \\
\text { or other individual captured? }\end{array}$ \\
\hline \multicolumn{2}{|c|}{ Characterisation of factor other than medical acuity } \\
\hline $\begin{array}{l}\text { General/collective } \\
\text { terminology }\end{array}$ & $\begin{array}{l}\text { What general or collective } \\
\text { terminology was used in this } \\
\text { study? }\end{array}$ \\
\hline $\begin{array}{l}\text { General/collective } \\
\text { definition }\end{array}$ & $\begin{array}{l}\text { What definition for the general/ } \\
\text { collective term was given by the } \\
\text { authors? }\end{array}$ \\
\hline $\begin{array}{l}\text { Individual factor } \\
\text { terminology }\end{array}$ & $\begin{array}{l}\text { What specific individual factors } \\
\text { were investigated? }\end{array}$ \\
\hline $\begin{array}{l}\text { Individual factor } \\
\text { definition }\end{array}$ & $\begin{array}{l}\text { What definition for the specific } \\
\text { individual factor was given by } \\
\text { the authors? }\end{array}$ \\
\hline \multicolumn{2}{|l|}{ Findings } \\
\hline Results & $\begin{array}{l}\text { What was the finding or result of } \\
\text { the study? }\end{array}$ \\
\hline
\end{tabular}

Team members serving as full-text data extractors will be trained together to ensure collective understanding of extraction data fields. As suggested by Daudt et $a l,{ }^{15} \mathrm{a}$ trial of independent data extraction will be performed to ensure consistency in data extraction and coding. A sample of the included studies will be reviewed by each full-text data extractor independently and compared for inter-rater reliability. If necessary, the data extraction template will be modified based on this pilot testing. As the scoping review is intended to be an iterative process, it is possible that additional themes will emerge and pertinent information will need to be added to the data extraction process. The research team will meet regularly to review progress and to discuss evolution of concepts. Any revisions to the data extraction process will be discussed collectively by all members of the research team.

While not a requirement of scoping reviews, the assessment of methodological quality is a hallmark of rigorous systematic reviews and will assist with the potential use of any findings that are reported. We will use the mixedmethods appraisal tool, which has been developed for complex systematic literature reviews such as this that combine qualitative, quantitative and mixed-methods studies. ${ }^{1617}$

\section{Stage 5: collating, summarising and reporting the results}

Information extracted in stage 4 will be summarised in tabular format. Quantitative analysis will be performed to produce descriptive statistics summarising the data contained in domains of study design and characterisation of factor other than medical acuity.

Because one of our central questions is how terminology is used in this literature, we will map terms used in each of the categories (collective/general and specific). Two reviewers will independently map terms used in the studies to a list we have created based on preliminary literature review (those referenced in the inclusion criteria: socioeconomic status, insurance status, barriers to healthcare access, lack of housing, food insecurity, mental health disorder, cognitive difficulties, physical disability or limitation, immigration status, non-English speakers). This list may be expanded for any terms encountered that do not map specifically. Results will be compared and discussed among the group until consensus is achieved and may be displayed graphically (if appropriate) or summarised in narrative form.

In addition, we intend to provide narrative summary in answer to the other subquestions listed in stage 1 above related to perspectives investigated, type of research performed and findings. Ideally, we also plan to construct a conceptual model for broader use, though it is possible that findings will not be able to be unified in this manner. Results reported in a final manuscript will follow recommendations included in the PRISMA Extension for Scoping Reviews (PRISMA-ScR) checklist. $^{18}$

\section{Stage 6: consultation}

Arksey and O'Malley suggest an optional final stage that includes consultation with stakeholders in order to add value and insight. Currently, we do not plan to formally include this step in the generation of a final product. Because this work has the potential to overlap with many aspects of research and clinical care, we anticipate that additional discussion and consultation will be completed with dissemination of the work at conferences and in publication. 


\section{PATIENT AND PUBLIC INVOLVEMENT}

There was no patient or public involvement in the preparation of this protocol. We have included extraction of data related to perspectives identified in the literature and plan to incorporate discussion of patient perspective within the literature as a whole.

\section{DISCUSSION}

The protocol describes a comprehensive approach that will survey current literature to elucidate existing information and reveal gaps in knowledge regarding the contributors other than medical acuity to hospitalisation. The final review product aims to summarise the current field of knowledge with respect to factors investigated, methods designed, findings identified and nomenclature used. This overview will better equip practitioners and policymakers to contextualise hospitalisation decisions and will provide researchers more complete understanding of the landscape of this field. This study addresses an urgent need for enhancing understanding and may inform new studies or policy. Understanding the impact of these factors on hospitalisation has potentially broad implications for quality improvement efforts, healthcare funding models and population or public health initiatives at local, state, national and international levels.

Contributors MB led the conceptualisation of the work. Both MH and MG contributed to the scope and design of the review as well as inclusion and exclusion criteria. MG prepared the manuscript for publication. MH designed the data extraction template. AM provided feedback on the methodology and crafted the search strategy. All authors contributed to editing and revising the manuscript and give approval for publication of this protocol.

Funding The authors have not declared a specific grant for this research from any funding agency in the public, commercial or not-for-profit sectors.

Competing interests None declared.

Patient consent for publication Not required.

Provenance and peer review Not commissioned; externally peer reviewed.

Open access This is an open access article distributed in accordance with the Creative Commons Attribution Non Commercial (CC BY-NC 4.0) license, which permits others to distribute, remix, adapt, build upon this work non-commercially, and license their derivative works on different terms, provided the original work is properly cited, appropriate credit is given, any changes made indicated, and the use is non-commercial. See: http://creativecommons.org/licenses/by-nc/4.0/.

\section{REFERENCES}

1. Dean NC, Jones JP, Aronsky D, et al. Hospital admission decision for patients with community-acquired pneumonia: variability among physicians in an emergency department. Ann Emerg Med 2012;59:35-41.

2. Jones B, Gundlapalli AV, Jones JP, et al. Admission decisions and outcomes of community-acquired pneumonia in the homeless population: a review of 172 patients in an urban setting. Am J Public Health 2013;103 Suppl 2(Suppl 2):S289-S293.

3. Rosenthal GE, Harper DL, Shah A, et al. A regional evaluation of variation in low-severity hospital admissions. J Gen Intern Med 1997;12:416-22.

4. Lewis Hunter AE, Spatz ES, Bernstein SL, et al. Factors influencing hospital admission of non-critically ill patients presenting to the emergency department: A cross-sectional study. J Gen Intern Med 2016;31:37-44.

5. O'Cathain A, Knowles E, Turner J, et al. Explaining variation in emergency admissions: a mixed-methods study of emergency and urgent care systems. Southamptom (UK): NIHR Journals Library 2014.

6. Calvillo-King L, Arnold D, Eubank KJ, et al. Impact of social factors on risk of readmission or mortality in pneumonia and heart failure: systematic review. J Gen Intern Med 2013;28:269-82.

7. Barnett ML, Hsu J, McWilliams JM. Patient characteristics and differences in hospital readmission rates. JAMA Intern Med 2015;175:1803-12.

8. Lin WC, Bharel M, Zhang J, et al. Frequent emergency department visits and hospitalizations among homeless people with medicaid: implications for medicaid expansion. Am J Public Health 2015;105 Suppl 5:S716-S722.

9. Doran KM, Ragins KT, lacomacci AL, et al. The revolving hospital door: hospital readmissions among patients who are homeless. Med Care 2013;51:767-73.

10. Chartier M, Carrico AW, Weiser SD, et al. Specific psychiatric correlates of acute care utilization among unstably housed HIVpositive adults. AIDS Care 2012;24:1514-8.

11. Weiser SD, Hatcher A, Frongillo EA, et al. Food insecurity is associated with greater acute care utilization among HIV-infected homeless and marginally housed individuals in San Francisco. J Gen Intern Med 2013;28:91-8.

12. Arksey H, O'Malley L. Scoping studies: towards a methodological framework. Int J Soc Res Methodol 2005;8:19-32.

13. Levac D, Colquhoun $\mathrm{H}$, O'Brien KK, et al. Scoping studies: advancing the methodology. Implement Sci 2010;5:69.

14. Moher D, Liberati A, Tetzlaff J, et al. Preferred reporting items for systematic reviews and meta-analyses: the PRISMA statement. PLoS Med 2009;6:e1000097.

15. Daudt HM, van Mossel C, Scott SJ. Enhancing the scoping study methodology: a large, inter-professional team's experience with Arksey and O'Malley's framework. BMC Med Res Methodol 2013;13:48.

16. Pluye P, Gagnon MP, Griffiths F, et al. A scoring system for appraising mixed methods research, and concomitantly appraising qualitative, quantitative and mixed methods primary studies in mixed studies reviews. Int J Nurs Stud 2009;46:529-46.

17. Pluye P, et al. Proposal: A mixed methods appraisal tool for systematic mixed studies reviews. 2011:(Accessed 7 Sep 2018). http://mixedmethodsappraisaltoolpublic.pbworks.com. Archived by WebCite at: http://www.webcitation.org/5tTRTc9yJ

18. Tricco AC, Lillie E, Zarin W, et al. PRISMA Extension for scoping reviews (PRISMA-ScR): Checklist and explanation. Ann Intern Med 2018;169:467-73. 